
\title{
\& Research Square \\ Frequency of Parechovirus Serotype 1 Among Young Infants With Sepsis
}

Manoochehr Makvandi ( $\sim$ manoochehrmakvandi299@gmail.com )

Ahvaz Jundishapur University of Medical Sciences

\section{Chiman Karami}

Ardabil University of Medical Sciences

\section{Rooya Pirmoradi}

Ahvaz Jondishapour University of Medical Sciences

\author{
Ali Timori \\ Hamedan University of Medical Science \\ Ahmad Shamsizadeh \\ Ahvaz Jondishapour University of Medical Sciences
}

\section{Research}

Keywords: Parchovirus, Sepsis, PCR( Polymerase Chain Reaction)

Posted Date: July 13th, 2020

DOI: https://doi.org/10.21203/rs.3.rs-39631/v1

License: (c) (1) This work is licensed under a Creative Commons Attribution 4.0 International License. Read Full License 


\section{Abstract}

Background: Human parechovirus $(\mathrm{HPeV})$ is recognized as a potentially severe viral infection such as gastrointestinal, respiratory and sepsis disease.In neonates and young infants. HPeV-1 is the most prevalent genotype and most commonly causes sepsis in young infants. The aim of study was to determine the prevalence of $\mathrm{HPeV}$ in hospitalized young infants with sepsis.

Methods: The sera of 100 samples were collected from young infants [46 (46\%) females and 54(54\%) males < below 90 days] with clinical signs and symptoms of sepsis. The total RNA was extracted, cDNA was synthesized. The Nested PCR was carried out for detection of HPeV. The mean , chi square tests were used for distributions of HPeV genotypes among the gender, age group and season.

Results: $5 / 100(5 \%)$ of patients including 2/46(2\%) females and 3/54(3\%) males showed positive for $\mathrm{HPeV}(P=0.85)$. The results of sequencing and phylogenetic tree revealed that the isolated HPeV were genotype 1.

Conclusion: Low prevalence of $5 \% \mathrm{HPeV}$ were detected. HPeV was dominant in this region. The screening HPeV RNA in patients with sepsis may reduce the use of antibiotics and shorten the duration of hospitalization.

\section{Introduction}

Human parechoviruses (HPeVs) are non-enveloped, single stranded RNA, positive-sense and belong to the family Picornaviridae (1). The HPeV genome is $\triangle 7350$ nucleotides ( $\mathrm{nt}$ ), comprised of structural protein (VP0, VP3 and VP1), seven non-structural proteins (2A, 2B, 2C, and 3A-3D) and two Untranslated Region regions; 5 UTR and 3 UTR (2).

HPeVs have been classified in the genus Parechovirus, which is divided into two species: Parechovirus $A$ and Parechovirus $B$. Parechovirus $A$ is classified into 19 genotypes, HPeV-1 to -19 , based on phylogenetic analysis of VP1 sequences, while Parechovirus $B$ comprises Ljungan viruses 1 to 4 genotypes (3-4). The prevalence of $\mathrm{HPeVs}$-A genotypic varies globally. HPeVs -A1 genotype is dominant in Europe and USA followed by PeV-A3 and PeV-A4, while PeV-A2, and A7-A19 genotypes are rarely reported (5). In Asia, similar to Europe and the USA, PeV-A1, A3, and A4 genotypes are the most prevalent and a higher diversity of genotypes has been reported in India and Pakistan $(6,7,8)$. In the African continent, PeV-A1, $\mathrm{A} 2$, and $\mathrm{A} 3$ are the most prevalent but nearly all HPeVs genotypes have been detected, indicating a much wider circulation of genotypes in this continent (9). The prevalence of HPeVs have been reported in young children with aseptic meningitis, sepsis-like illness and gastroenteritis in Iran (10-12)

PeV-A1 has been detected in acute gastroenteritis, upper respiratory tract symptoms, fever, rash ,paralysis and encephalitis in children age 6 months to 5 years (13-16). 
$\mathrm{HPeV} 3$, was recognized in children with respiratory, gastroenteritis and sepsis infections (5). HPeV-3 is the most important agent for asepsis, sepsis-like illness and CNS infection in infants > 3 months (17-20). $\mathrm{HPeV}-4$ has been identified in patients with nervous system infection (21). HPeV-6 has been reported from a 1-year old fatally child with Reye syndrome (22) and isolated from a stool sample of a 2.5-monthold girl with fever, dehydration, bilateral otitis media, and anemia (23).

$\mathrm{HPeVs}$ can be Transmitted via the fecal-oral route and the respiratory tract $(24,25)$. At present There is no standard approach for diagnosis of viral sepsis but molecular approach such as PCR provides greater sensitivity and specificity than DFA and cell culture methods (26).

Serum samples are the most useful compared to CSF for detection of HPeV RNA from neonates and young infants with sepsis and encephalitis (27). Early recognition of HPeV infections is very important since it may reduce the use of antibiotics and shorten the duration of hospital admissions for neonates and young infants with sepsis (28).

This study was conducted to evaluate the frequency of HPeV genotype in hospitalized young infant with clinical sign and symptoms of sepsis like syndrome in Ahvaz city, Iran

\section{Materials And Methods}

In this prospective cross sectional study, 100 sera were collected from young infants [46 (46\%) females and 54(54\%) males < below 90 days] who had been admitted with clinical signs and symptoms of sepsis at the Abozar Children's Hospital, Ahvaz city, Iran during Sept 2016 until October 2017.The Exclusion criteria were congenital anomalies (including cerebral malformations) and positive for bacterial culture.

The patients ages were between 20-90 days with mean age of $54 \pm 22$ who were admitted to the intensive care unit with sepsis. The definition of sepsis was based on the following criteria ; Fever $>38^{\circ} \mathrm{C}$, Poor feeding (76\%), Lethargy(47\%), irritability(97\%),Tachypnea(78\%),Tachycardia(91\%),rash(67\%),vomiting(27\%),diarrhea(41\%), Poor Perfusion(54\%), apnea (12\%). The analyses of proteins, CBC and serological tests such as C-reactive were carried out for all the participants.

\section{Statistical analysis}

Data analysis was performed using the Statistical Package for the Social Sciences 22.0 (SPSS Inc., Chicago, IL, USA). Chi-Square test was used to calculate. $\mathrm{P}<0.05$ was accepted as significant.

\section{Extraction of RNA and cDNA synthesis}

The total RNA was extracted from each serum sample using high pure viral nucleic acid( Roche company, Germany) according to instructor manufacture. The cDNA was prepared using (Thermo scientific, USA) company, according to manufacture instructor. 


\section{Nested PCR}

The Nested-PCR was carried using the following outer primers, forward: AN353( 2126-2154) 5'GACAATAGTTTTGAAATNACNATHCCNTA- 3', , and reverse: AN358 (3116-3086) 5'AACTATAATGCCATARTGYT TRTARAANCC- $3^{\prime}$, and inner primers, forward: AN369 ( 2531-2559) 5' ACCAAGGTTGACAACATTTTYGGNMGNGC - 3', and inner primers, reverse AN357( 2829-2798), 5'GAATAAAATGGTACTGANARNG TCATYTGYTC - 3', (21). First round PCR was performed with $25 \mu \mathrm{l}$

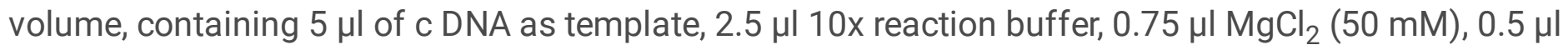
$(10 \mu \mathrm{M})$ forward/reverse primer, $1 \mu \mathrm{l}$ dNTP $(10 \mathrm{mM}), 0.2$ Cinna Gen Taq DNA Polymerase $(5 \mathrm{u} / \mu \mathrm{l})$, and $13.55 \mu \mathrm{l}$ double-distilled water. The reaction mixture was subjected to thermal cycler (TC-512, Techne, Staffordshire, UK) with the following program: 1 cycle with initial denaturation at $95^{\circ} \mathrm{C}$ for 5 minutes, followed by 35 cycles, $94{ }^{\circ} \mathrm{C}$ for $30 \mathrm{~s}, 42^{\circ} \mathrm{C}$ for $40 \mathrm{~s}, 72{ }^{\circ} \mathrm{C}$ for $60 \mathrm{~s}$, and final extension at $72{ }^{\circ} \mathrm{C}$ for 3 minutes. $1 \mu \mathrm{l}$ of PCR product of the first round of was used as the template for the second round. The amount of PCR components was the same as the first round. The thermal cycler was programmed as follows: initial denaturation at $95^{\circ} \mathrm{C}$ for 5 minutes, 35 cycles consisting of $95^{\circ} \mathrm{C}$ for $30 \mathrm{~s}, 58{ }^{\circ} \mathrm{C}$ for $30 \mathrm{~s}$, $72{ }^{\circ} \mathrm{C}$ for $30 \mathrm{~s}$, and final extension at $72{ }^{\circ} \mathrm{C}$ for 3 minutes. All reactions were performed in the presence of negative and positive controls. The PCR product of 293 bp shows the positive reaction (29).

\section{Sequencing Of Isolated Hbv Dna}

The results of two PCR products were sequenced using ABI 3730xI DNA sequencer (Applied Biosystems, USA). The partial sequences of parechovirus genome was sent to Gene Bank to obtain the accession number.

\section{Phylogenetic tree}

The phylogenetic tree was constructed by Neighbor joining method for partial VP1 region of two isolated $\mathrm{HPeV}$ and compared with different $\mathrm{HPeV}$ genotypes retrieved from GeneBanks. The Neighbor joining was built under Kimura two-parameter model, with reproducibility of 1,000 of bootstrap and scale bare $=$ 0.005 .

\section{Results:}

$46 / 100(46 \%)$ of the patients were females and $54(54 \%)$ males $<90$ days with a mean age of $67 \pm 22$ days. The sera of $5(5 \%)$ samples [ 2/46 females and 3/54 males] were positive for HPeV RNA by Nested PCR. ( $P=0.85)$. The patients profile present in the table 1 . The incidence of HPeVe among the young neonates with sepsis were taken place in the $2(2 \%)$ in autumn and $3(3 \%)$ winter season. No HPeVe detection was found in the summer and spring seasons.

Table 2.Profile of neonates sepsis patients associated with HPeV. 

Characteristic
$\mathrm{HPeV}$ positive
$\%$
$P$ value

No. of patients (100)

5

5

Sex

Female

$2 / 46$

4.34

0.85

Males

$3 / 54$

5.55

\section{Age groups(days)}

$1-19$

20-29

30-90

Season

Spring

Summer

Autumn

Winter

\section{Laboratory findings}

$\operatorname{WBC}\left(\mathrm{mm}^{3}\right)$

NA

9990-22000

Absolute neutrophil count $\left(\mathrm{mm}^{3}\right)$

NA

Platelet $\left(\times 10^{3} / \mathrm{mm}^{3}\right)$

$3 / 68$

4.41
$-/ 19$

2/41

$3 / 40$

6.25

0.87

0.48

7.5 
The sequences of two isolated HPeVes were deposited in GenBank with accession numbers, MN845069 and MN845070. Both isolated HPeVes showed 97\% nucleotide identity with HPeV genotype1( MK792785.1) isolated from Tehran, Iran.

\section{Phylogenetic Tree}

The analysis of phylogenetic tree revealed that both the isolated HPeV (MN845069 and MN845070) are cluster with HPeVes serotype 1 isolated from different regions of the world (Figure A).

\section{Discussion}

Sepsis due to HPeV may result in notable morbidity in neonates and young children. Although, most of $\mathrm{HPeV}$ infections are self-limited, but long-term neurological deficits such as learning disability, paralysis, epilepsy and encephalitis have been reported in patients with HPeV infections $(16-22,30)$. It has been repoted the prevalence of HPeV3 is much more associated with sepsis disease than HPeV1 (17-20).

In the present survey, the prevalence of HPeV1 was 5/100 (5\%) [2(2\%) Females and 3(3\%) males] in young infants with sepsis $(P=0.85)$. No HPeV3 was detected in the patients. The studies have shown $\mathrm{HPeV} 1$ was dominant in young children with aseptic meningitis and sepsis-like illness and gastroenteritis In Iran (10-12). The results of sequencing of two VP1 region of isolated HPeV1 (MN845069 and MN845070) showed $97 \%$ nucleotide identity with HPeV genotype 1 isolated from Tehran, Iran ( MK792785.1). The results of phylogenic tree of two isolated HPeV1 (MN845069 and MN845070) were cluster with HPeV1 isolated from different regions of the world. In this study, the incidence of HPeV1 was found in $2(2 \%)$ cases in autumn and $3(3 \%)$ cases winter season. No HPeV1 detection was detected in summer and spring seasons.

Rahimi et al, (2013) have detected HPeV 1 infections throughout the years with no significant difference in infection rate between seasons. However, the most HPeV 1 detections were found during summer and the lowest HPeV detection rate was in spring in Tehran ,Iran (10).

In a multinational epidemiological study have been described the etiology of one-third children with severe sepsis, were viral infections (31). The most frequent sites of infection were the respiratory tract (40\%) with rhinovirus, Respiratory syncytial virus, and adenovirus most commonly isolated in children with sepsis (31). In Australia and New Zealand the etiology of $50 \%$ of patients with sepsis were RSV, HVR, CMV, EBV, HSV, VZV

and influenza (32). The previous study have reported that $15 \%$ of neonates with sepsis, were bacterial etiology (33).

In the present study the role of other viruses such as human rhinovirus, human Respiratory syncytial virus, human Enterovirus (EV), HAvd, CMV, EBV, HSV, VZV and influenza have not been investigated in patients with sepsis, but it requires comprehensive investigation. 
To date, no antiviral drug has been shown to be effective against HPeV, and no vaccines are currently available to protect against infection. The IVIGs have been used for the treatment of severe disease. An infant with severe, dilated cardiomyopathy caused by HPeV-1 was fully recovered after treatment with Intravenous immunoglobulin) IVIG (34).

Although low detection of $5 \% \mathrm{HPeV}$ were found among young infant but the screening of HPeV RNA should be implemented in young infants with sepsis. The implementation of molecular detection of $\mathrm{HPeV}$ is very pivotal as it reduce the use of antibiotics and shorten the duration of hospitalization. the role of other viruses such as HRV, RSV, and HAdV, HCMV, EBV, HSV, VZV and influenza have not been investigated in patients with sepsis, but it requires comprehensive investigation.

\section{Conclusion}

The main goal of the current study was to determine Frequency of Parechovirus serotype 1 among young infants with sepsis. This study has identified low prevalence of $5 \% \mathrm{HPeV}$ were detected. HPeV was dominant in this region. The screening HPeV RNA in patients with sepsis may reduce the use of antibiotics and shorten the duration of hospitalization. Further studies need to be carried out in order to validate these finding.

\section{Abbreviations}

Human parechovirus HPeV, Untranslated Region UTR, Polymerase Chain Reaction PCR, Direct Fluorescent Antibody DFA, human respiratory syncytial virus RSV, human Enterovirus EV, human adenovirus HAdV, HVR, human cytomegalovirus CMV, Epstein-Barr virus EBV, herpes simplex virus HSV, varicella zoster virus VZV, Intravenous immunoglobulin IVIG.

\section{Declarations}

\section{Ethic consent}

The project was approved by ethic committee of Ahvaz Jundishapur university of Medical Sciences, Ahvaz Iran. The informed consent was obtained from all the parents-neonates who were participated in this study.

\section{Acknowledgment}

This project with the registration number OG-95108 was recorded by Infectious and Tropical Diseases Research Center, Health Research Institute, Ahvaz Jundishapur University of Medical Sciences, Ahvaz, Iran

\section{Authors' contributions}

Authors' contributions $\mathrm{M} \mathrm{M}$, conceptualized and designed the study, reviewed and revised the manuscript and approved the final manuscript as submitted. $\mathrm{CH} \mathrm{K}$, carried out the data collection, analyses, reviewed and revised the manuscript and approved the final manuscript as submitted. $\mathrm{CH} \mathrm{K}$, carried out the data 
collection and analyses. R P, carried out the data collection and analyses. A SH carried out the data collection and analyses. A T , All authors read and approved the final manuscript

\section{Consent for publication}

"Not applicable"

\section{Availability of data and materials}

"Not applicable"

\section{Authors' information}

1 Infectious and Tropical Diseases Research Center, Health Research Institute, Ahvaz Jundishapur University of Medical Sciences, Ahvaz, Iran

2 Virology Department, School of Medicine, Ahvaz Jundishapur University of Medical Sciences, Ahvaz, IR Iran

3 Pediatric Infectious Division, Abuzar pediatrics hospital, Ahvaz Jundishapur University of Medical Sciences, Ahvaz, Iran

${ }^{*}$ Corresponding author: Manoochehr Makvandi, Infectious and Tropical Diseases Research Center, Health Research Institute, Ahvaz Jundishapur University of Medical Sciences, Ahvaz, Iran IR Iran. Tel: +989166181683, Fax: +98-6113738313, E-mail: manoochehrmakvandi299@gmail.com

\section{Competing interests}

No Competing interests

\section{Funding}

This project was financially supported by Infectious and Tropical Diseases Research Center, Health Research Institute, Ahvaz Jundishapur University of Medical Sciences, Ahvaz, Iran

\section{References}

1-Tapparel C, Siegrist F, Petty TJ, Kaiser L. 2013. Picornavirus and enterovirus diversity with associated human diseases. Infect Genet Evol 14:282-293

2-G. Stanway, P. Joki-Korpela, T. Hyypia, Human HPeVes-biology and clinical significance, Rev. Med. Virol. 10 (2000) 57-69.

3-. Zhao X, Shi Y, Xia Y. 2016. Genome analysis revealed novel genotypes and recombination of the human HPeVes prevalent in children in Eastern China. Gut Pathog 8:52.

4- Olijve L, Jennings L, Walls T. 2018. Human HPeV: an increasingly recognized cause of sepsis-like illness in young infants. Clin Microbiol Rev 31:e00047-17. https://doi.org/10.1128/CMR.00047-17.

5- Adithya Sridhar , Eveliina Karelehto , Lieke Brouwer , Dasja Pajkrt, Katja C. Wolthers. HPeV A Pathogenesis and the Enigma of Genotype A-3. Viruses 2019, 11, 1062; doi:10.3390/v11111062 
6-Zhong, H.; Lin, Y.; Sun, J.; Su, L.; Cao, L.; Yang, Y.; Xu, J. Prevalence and genotypes of human HPeV in stool samples from hospitalized children in Shanghai, China, 2008 and 2009. J. Med. Virol. 2011, 83, 1428-1434

7-Alam, M.M.; Khurshid, A.; Shaukat, S.; Rana, M.S.; Sharif, S.; Angez, M.; Nisar, N.; Aamir, U.B.; Naeem, M.; Zaidi, S.S. Viral etiologies of acute dehydrating gastroenteritis in pakistani children: Confounding role of HPeVes. Viruses 2015, 7, 378-393.

8-Patil, P.R.; Ganorkar, N.N.; Gopalkrishna, V. Epidemiology and genetic diversity of human HPeVes circulating among children hospitalised with acute gastroenteritis in Pune, Western India: A 5-years study. Epidemiol. Infect. 2018, 146, 11-18.

9-Graul, S.; Bottcher, S.; Eibach, D.; Krumkamp, R.; Kasmaier, J.; Adu-Sarkodie, Y.; May, J.; Tannich, E.; Panning, M. High diversity of human HPeV including novel types in stool samples from Ghanaian children. J. Clin. Virol. 2017, 96, 116-119

10-Rahimi P, Naser HM, Siadat SD, Sohrabi A, Mostafavi E, Motamedirad M, Bahramali G, Sadat SM, Ardestani MS.Genotyping of human HPeVes in Iranian young children with aseptic meningitis and sepsis-like illness. J Neurovirol. 2013 Dec;19(6):595-600. doi: 10.1007/s13365-013-0221-7.

11-Shokrollahi MR, Noorbakhsh S, Monavari HR, Darestani SG, Motlagh AV, Javadi Nia S.Acute Nonbacterial Gastroenteritis in Hospitalized Children: A Cross Sectional Study. Jundishapur J Microbiol. 2014 December; 7(12): e11840. DOI: 10.5812/jjm.11840

12- Ghazi F, Ataei Z , Dabirmanesh B. Molecular detection of human HPeV type 1 in stool samples from children with diarrhea. International Journal of Infectious Diseases 16 (2012) e673-e676

13- Ito, M.; Yamashita, T.; Tsuzuki, H.; Kabashima, Y.; Hasegawa, A.; Nagaya, S.; Kawaguchi, M.; Kobayashi, S.; Fujiura, A.; Sakae, K.; et al. Detection of human HPeVes from clinical stool samples in Aichi, Japan. J. Clin. Microbiol. 2010, 48, 2683-2688.

14-Chiang, G.P.K.; Chen, Z.; Chan, M.C.W.; Lee, S.H.M.; Kwok, A.K.; Yeung, A.C.M.; Nelson, E.A.S.; Hon, K.L.; Leung, T.F.; Chan, P.K.S. Clinical features and seasonality of HPeV infection in an Asian subtropical city, Hong Kong. PLoS ONE 2017, 12, e0184533.

15-Ito, M.; Yamashita, T.; Tsuzuki, H.; Takeda, N.; Sakae, K. Isolation and identification of a novel human HPeV. J. Gen. Virol. 2004, 85, 391-398

16-Verboon-Maciolek, M.A.; Groenendaal, F.; Hahn, C.D.; Hellmann, J.; van Loon, A.M.; Boivin, G.; de Vries, L.S. Human HPeV causes encephalitis with white matter injury in neonates. Ann. Neurol. 2008, 64, 266273

17- Levorson RE, Jantausch BA, Wiedermann BL, Spiegel HM, Campos JM. 2009. Human HPeV-3 infection: emerging pathogen in neonatal sepsis. Pediatr Infect Dis J 28:545-547. 
18- Olijve L, Jennings L, Walls T. 2018. Human HPeV: an increasingly recognized cause of sepsis-like illness in young infants. Clin Microbiol Rev 31:e00047-17.

19- Wolthers KC, Benschop KS, Schinkel J, Molenkamp R, Bergevoet RM, Spijkerman IJ, Kraakman HC, Pajkrt D. 2008. Human HPeVes as an important viral cause of sepsis like illness and meningitis in young children. Clin Infect Dis 47:358 -363

20-Harvala H, Robertson I, Chieochansin T, McWilliam Leitch EC, Templeton K, Simmonds P. 2009.

Specific association of human HPeV type 3 with sepsis and fever in young infants, as identified by direct typing of cerebrospinal fluid samples. J Infect Dis 199:1753-1760. https://doi.org/10.1086/599094.

21- Sasidharan A, Harrison CJ, Banerjee D, Selvarangan R. 2019. Emergence of HPeV A4 central nervous system infections among infants in Kansas City, Missouri, USA. J Clin Microbiol 57:e01698-18

22- Watanabe K, Oie M, Higuchi M, Nishikawa M, Fujii M.Isolation and characterization of novel human HPeV from clinical samples. Emerg Infect Dis. 2007 Jun; 13(6):889-95.

23-de Vries $M^{1}$, Pyrc K, Berkhout R, Vermeulen-Oost W, Dijkman R, Jebbink MF, Bruisten S, Berkhout B, van der Hoek LHuman HPeV type 1, 3, 4, 5, and 6 detection in picornavirus cultures. J Clin Microbiol. 2008 Feb;46(2):759-62

24- Wildenbeest JG, Benschop KS, Bouma-de Jongh S, Wolthers KC, Pajkrt D. 2016. Prolonged shedding of human HPeV in feces of young children after symptomatic infection. Pediatr Infect Dis J 35:580 583.

25- Sharp J, Bell J, Harrison CJ, Nix WA, Oberste MS, Selvarangan R. 2012. Human HPeV in respiratory specimens from children in Kansas City, Missouri. J Clin Microbiol 50:4111- 4113.

26- Gupta N, Richter R, Robert S and Kong M (2018) Viral Sepsis in Children. Front. Pediatr. 6:252. doi: 10.3389/fped.2018.00252

27- Aizawa Y, Suzuki Y, Watanabe K, Oishi T, Saitoh A. Clinical utility of serum samples for human HPeV type 3 infection in neonates and young infants: the 2014 epidemic in Japan. J Infect 2016, 72:223-232. https://doi.org/10.1016/j.jinf.2015.10.010.

28- Kari A. Simonsen, a Ann L. Anderson-Berry, b Shirley F. Delair, a H. Dele Daviesa. Early-Onset Neonatal Sepsis. Clinical Microbiology Reviews. 2014, 27( 1); 21- 47

29- Nix WA, Maher K, Pallansch MA, Oberste MS. 2010. HPeV typing in clinical specimens by nested or semi-nested VP1 PCR coupled with sequencing. J. Clin. Virol. 48:202-207.

30- Khatami A, McMullan BJ, Webber M, Stewart P, Francis S, Timmers KJ, Rodas E, Druce J, Mehta B, Sloggett NA, Cumming G, Papadakis G, Kesson AM. 2015. Sepsis-like disease in infants due to human 
HPeV type 3 during an outbreak in Australia. Clin Infect Dis 60:228 -236.

https://doi.org/10.1093/cid/ciu784.

31- Weiss SL, Fitzgerald JC, Pappachan J, Wheeler D, Jaramillo-Bustamante JC, Salloo A, et al. Global epidemiology of pediatric severe sepsis: the sepsis prevalence, outcomes, and therapies study. Am J Respir Crit Care Med. (2015) 191:1147-57. doi: 10.1164/rccm.201412-23230C

32-Schlapbach LJ, Straney L, Alexander J, MacLaren G, Festa M, Schibler A, et al. Mortality related to invasive infections, sepsis, and septic shock in critically ill children in Australia and New Zealand, 200213: a multicenter retrospective cohort study. Lancet Infect Dis. (2015) 15:46-54. doi: 10.1016/S14733099(14)71003-5

33- Byington CL, Enriquez FR, Hoff C, Tuohy R, Taggart EW, Hillyard DR, et al. Serious bacterial infections in febrile infants 1 to 90 days old with and without viral infections. Pediatrics (2004) 113:1662-6.

34- Wildenbeest JG, Wolthers KC, Straver B, Pajkrt D. 2013. Successful IVIG treatment of human HPeVassociated dilated cardiomyopathy in an infant. Pediatrics 132:e243- e247.

\section{Figures}




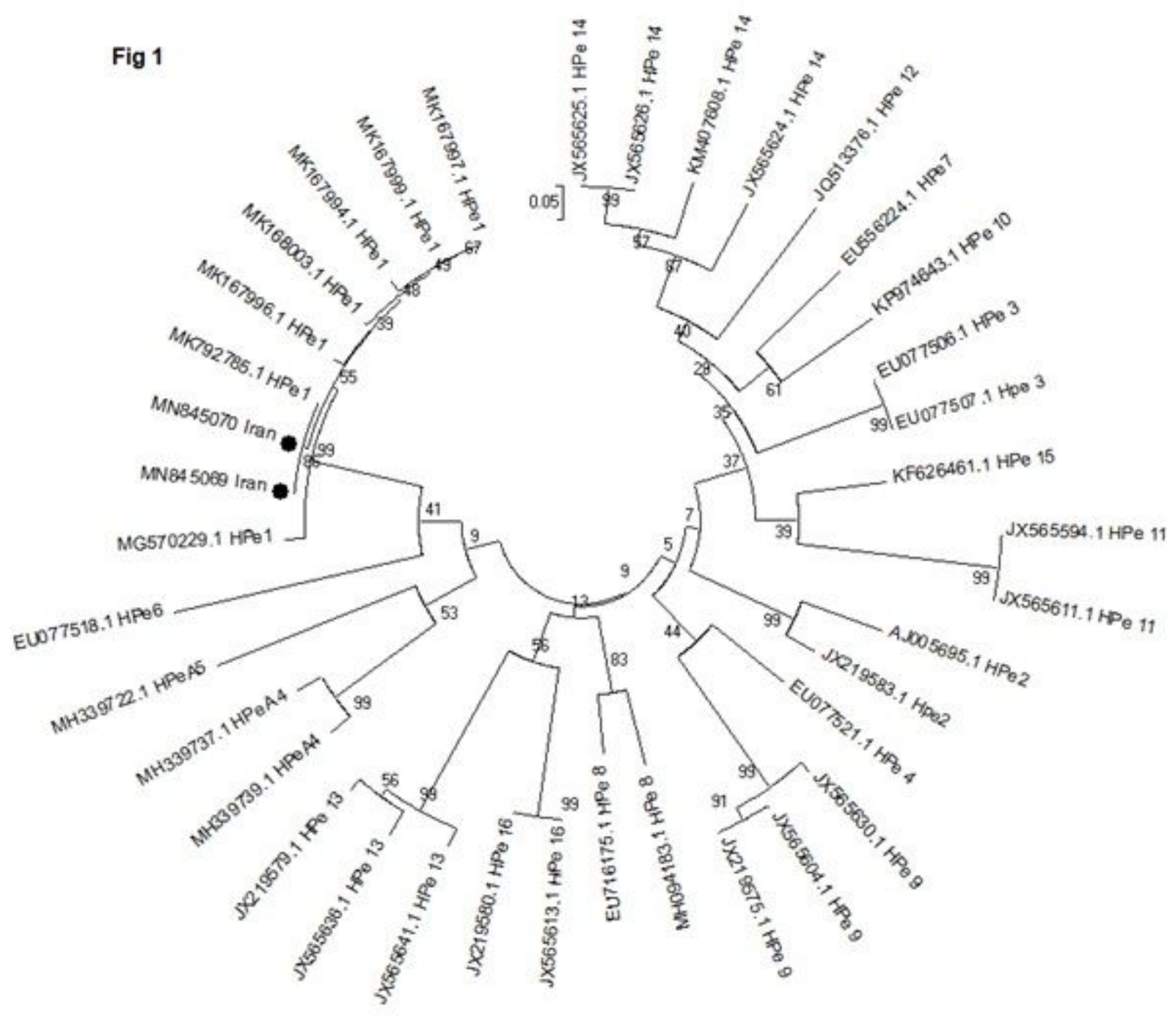

\section{Figure 1}

A phylogenic tree was constructed with Neighbor joining method for partial VP1 region of two isolated $\mathrm{HPeV}$ from two patients with sepsis in Ahvaz city. The two isolated HPeV RNA with accession numbers (MN845069, MN845070) were compared with the different HPeVes genotypes retrieved from GenBank. The results of phylogenetic revealed that the Iranian isolates with black circle color are cluster with $\mathrm{HPeV}$ genotype 1 isolatedfrom different regions of the world. The accuracy was assessed by 1000 bootstrap replicates. Scale bar $=0.005$ 